\title{
Over-expression of EBP50 supresses the invasion and migration of CIA-FLS in vitro by downregulating MMP-9
}

Jie Zhou ${ }^{1}$, yu gao ${ }^{2}$, Chaofei $\mathrm{Tu}^{3}$, HuaPing Zhou ${ }^{4}$, ri su ${ }^{2}$, zi huang ${ }^{3}$, Junming $\mathrm{Li}^{5}$, qing luo ${ }^{6}$, and yang guo ${ }^{3}$

${ }^{1}$ Maternal and Child Health Hospital of Jiangxi Province

${ }^{2}$ Nanchang University

${ }^{3}$ The First Affiliated Hospital of Nanchang University

${ }^{4}$ People's Hospital of Jiangxi Province

${ }^{5}$ the First Affiliated Hospital of Nanchang University

${ }^{6}$ The First Affiliated Hospital Of Nanchang University

October 1, 2020

\begin{abstract}
Studies had confirmed that the abnormal proliferation and activation of FLS play a key role in the process of arthropathy. Inhibiting the abnormal proliferation and activation of FLS may be an effective method to control the development of RA. Many studies showed that EBP50 was a powerful factor in inhibiting the abnormal tumor cell proliferation and activation, but the expression characteristics and function of EBP50 in FLS had not yet been reported. In this study we showed that overexpression of EBP50 in CIA-FLS can inhibit the proliferation, which had no effect on the autophagy, and promoted the cell apoptosis by activating caspase-3. At the same time, relative results revealed that EBP50 overexpression markedly down-regulated the expression of MMP-9, but had no effect on MMP-2, E-cadherin and $\beta$-catenin, suggesting that one of the mechanisms for EBP50-regulated aggressive behavior of FLS is reducing MMP-9 production.
\end{abstract}

Title Page

Title

Over-expression of EBP50 supresses the invasion and migration of CIA-FLS in vitro by downregulating MMP-9

Full names

Jie Zhou ${ }^{1,2^{*}}$, Yujie Gao ${ }^{3 *}$, Chaofei $\mathrm{Tu}^{1}$, Huaping Zhou ${ }^{4}$, Rigu Su${ }^{1}, Z i k u n$ Huang ${ }^{1}$, Junming Li $^{1 \#}$, Qing Luo ${ }^{1 \#}$ and Yang Guo ${ }^{1 \#}$

Institutional addresses

1 Department of Clinical Laboratory, The First Affiliated Hospital of Nanchang University, Nanchang, Jiangxi 330006, China.

2 Department of Clinical Laboratory, Maternal and Child Health Hospital of Jiangxi Province,Nanchang, Jiangxi 330006, China.

3 Department of medical college, Nanchang University, Nanchang, Jiangxi 330006,China. 
4 Department of Clinical Laboratory, People's Hospital of Jiangxi Province, Nanchang, Jiangxi 330006, China.

Email addresses for all authors

Jie Zhou: 352346483@qq.com

Yujie Gao: 1024608270@qq.com

Chaofei Tu: 247930460@qq.com

Huaping Zhou: 879123144@qq.com

Rigu Su: 851242097@qq.com

Zikun Huang: 491353062@qq.com

Junming Li: lisir361@163.com

Qing Luo: lxc042@163.com

Yang Guo: 839444295@qq.com

*equally contributed to this study

\#Corresponding Author

Dr. Junming Li. Department of Clinical Laboratory, First Affiliated Hospital of Nanchang University, Nanchang, Jiangxi 330006, China. Tel: (+86) 0791-88692794. Fax: (+86) 0791-88623153. E-mail: lisir361@163.com

Qing Luo. Department of Clinical Laboratory, First Affiliated Hospital of Nanchang University, Nanchang, Jiangxi 330006, China. Tel: (+86) 0791-88692794. Fax: (+86) 0791-88623153. E-mail: lxc042@163.com

Yang Guo. Department of Clinical Laboratory, First Affiliated Hospital of Nanchang University, Nanchang, Jiangxi 330006, China. Tel: (+86) 0791-88697032. Fax: (+86) 0791-88623153. E-mail: 839444295@qq.com

\section{Absract}

Studies had confirmed that the abnormal proliferation and activation of FLS play a key role in the process of arthropathy. Inhibiting the abnormal proliferation and activation of FLS may be an effective method to control the development of RA. Many studies showed that EBP50 was a powerful factor in inhibiting the abnormal tumor cell proliferation and activation, but the expression characteristics and function of EBP50 in FLS had not yet been reported. In this study we showed that overexpression of EBP50 in CIA-FLS can inhibit the proliferation, which had no effect on the autophagy, and promoted the cell apoptosis by activating caspase-3. At the same time, relative results revealed that EBP50 overexpression markedly down-regulated the expression of MMP-9, but had no effect on MMP-2, E-cadherin and $\beta$-catenin, suggesting that one of the mechanisms for EBP50-regulated aggressive behavior of FLS is reducing MMP-9 production.

Keywords: EBP50, FLS, Rheumatoid arthritis

\section{Introduction}

Rheumatoid arthritis (RA) is a chronic, progressive autoimmune disease characterized by aggressive and symmetric polyarthritis ${ }^{[1,2]}$. The principal pathological changes in RA include chronic persistent synovial inflammation, abnormal proliferation of synovial cells, extensive infiltration of inflammatory cells, formation of synovial membrane pannus, gradually eroded cartilage and subchondral bone, then resulting in destruction of joint tissue structure and loss of function ${ }^{[3,4]}$. RA has been reported to occured in people aged 40 to 60 , while showing predominance in women more than men. Although current clinical treatment can alleviate some symptoms, it cannot reverse the damage caused to cartilage and related bone tissue, and the high disability rate of RA seriously affects the quality of patients life. The latest research have shown that the 
occurrence and development of RA is due to a combination of genetic, environmental and immune factors, but the specific mechanism is still unclear ${ }^{[5]}$.

Fibroblast-like synoviocytes (FLS) are predominant component of joint synovial membrane ${ }^{[6]}$, and plays an pivotal physiological role in controlling the volume of synovial fluid and maintaining the stability of joint cavity $^{[7]}$. At the same time, FLS are also important factor leading to joint pathological damage ${ }^{[8]}$. During the occurrence and development of RA, FLS can occur three major abnormal changes: such as releasing large amounts of proinflammatory cytokines ${ }^{[9]}$, FLS can promote the occurrence and maintenance of inflammatory response by involving in the activation of macrophages, T lymphocytes and NK cells ${ }^{[10]}$; such as dysplasia and apoptosis defect, both proliferation and invasion of FLS were significantly enhanced ${ }^{[11]}$, exhibiting tumor-like behavior, accompanied by significant inhibition of apoptosis ${ }^{[2]}$; such as secreting matrix degrading enzymes, including matrix apoptosis-deficient metalloproteinases and cathepsin,leading to destruction of bone and cartilage $^{[13,14]}$. A large number of studies have confirmed that abnormal proliferation and activation of FLS play a key role in the process of joint lesions in RA patients ${ }^{[15,16]}$. Therefore, inhibiting abnormal proliferation and activation of FLS may be a potentially therapeutic strategy to block or delay the progression of RA.

EBP50, also known as $\mathrm{Na}+\mathrm{H}+$ exchange regulatory factor (NHERF1), is a PSD-95/Dlg-1, Drosophila disk large/ZO-1 (PDZ)-containing scaffolding protein that regulates a variety of physiological functions, such as controlling the localization, delivery, surface stability and function of transporters, integral membrane proteins, and ion channel proteins, as well as clustering of proteins to specific cellular domains to facilitate signaling. As a novel tumor suppressor gene. Previous studies have demonstrated that EBP50 expression was down-regulated in breast cancer ${ }^{[17]}$, gastric cancer ${ }^{[18]}$ and prostate cancer ${ }^{[19]}$, and overexpression of EBP50 could effectively inhibit the proliferation of tumor cells and promote the apoptosis of tumor cells, at the same time, Related researchs also show that EBP50 can inhibit the migration and invasion of breast cancer cells by down-regulating MMPs and interacting with $\beta$-catenin ${ }^{[20,21]}$.

However, most studies of EBP50 have focused on tumor cells, and it is not clear whether EBP50 can perform the same function in other cells. In this study, we investigated the effects and mechanisms of EBP50 overexpression on the Proliferation and Migration of Fibroblast-Like Synoviocytes in vitro.

\section{Materials and Methods}

Materials. Antibodies specific for EBP50 (ab3452), MMP-2 (ab37150), MMP-9 (ab38898) were purchased from Abcam (San Francisco, CA); Caspase-3 (9662), LC-3 (4108), $\beta$-catenin (8480) and E-cadherin(14472) were purchased from CST (Cell Signaling Technology); $\beta$-actin mAb (AC062),HRP Goat Anti-Rabbit IgG (AS014) and HRP Goat Anti-Mouse IgG (AS003) was purchased from ABclonal Technology; The CIAFLS were kindly gifted by professor Yingyuan Fu from the Department of Immunology, Medical College of Nanchang University, Nanchang, China, The lentiviral expression plasmid pLVX-IRES-ZsGreen1 was kindly gifted by ZhangLin Zhang from the Department of Transfusion, First Affiliated Hospital of Nanchang University, Nanchang, Jiangxi, China.

Cell culture. The CIA-FLS were maintained in Dulbecco's modified Eagle medium (DMEM, Sigma-Aldrich) supplemented with $10 \%$ fetal bovine serum (FBS). 293T cells (ATCC, TIB-202) were maintained in RPMI 1640 medium (GIBCO, Grand Island, NY) supplemented with 10\% FBS. The cells were cultured at 37 with an atmosphere of $5 \% \mathrm{CO}_{2}$ and $95 \%$ air.

Lentivirus vector construction. The EBP50 encoding gene was amplified from RAW264.7 cells by reversetranscription-polymerase chain reaction (RT-PCR). The primers containing EcoRI and BamHI sites for PCR were as follows, forward primer: 5'-AGAGAATTC-ATGAGCGCGGACG-3'; reverse primer: 5'CGCGGATCC-TCAGAGGTTGCTGAAGA-3'. The thermal cycling condition for PCR was 95 for 5 min, 30 cycles at 94 for $40 \mathrm{~s}, 54$ for $30 \mathrm{~s}$ and 72 for $90 \mathrm{~s}$, followed by a final round of 72 for $10 \mathrm{~min}$. The PCR product was digested by EcoRI and BamHI, and ligated with linearized Plvx-Ires-ZsGreen1 by T4 DNA ligase to form recombinant lentiviral expression vector Plvx-EBP50. 
Lentiviral vectors package. 293T cells were cultured into 6 -well plates at $37{ }^{\circ} \mathrm{C}$ with $5 \% \mathrm{CO} 2$ in humidified atmosphere. After reaching $70 \% \sim 80 \%$ confluence, $293 \mathrm{~T}$ cells were triple transfected with the lentiviral expression vector Plvx-EBP50 and two packaging vectors pSPAX2 and pMD2.G as a group named LV-EBP50, and Plvx-Ires-ZsGreen1, pSPAX2 with pMD2.G as a group named LV-Plvx by using FuGene 6 transfect regent following the manufacturers protocol. Culture supernatants were collected every $24 \mathrm{~h}$ for 3 days, filtered through a $0.45-\mu \mathrm{m}$ poresize filter, and concentrated two times with ultracentrifugation at $50,000 \times \mathrm{g}$ for 120min. Each group was diluted 1:10, 1:100, and 1:1000 times to determine virus titers. The virus particles were resuspended in sterile PBS, and stored at $-80{ }^{\circ} \mathrm{C}$ until use.

Real-time PCR. Total cellular RNA was extracted from cells using TRIzol reagent (Invitrogen, Carlsbad, $\mathrm{CA}$ ), following the manufacturer's instruction, then reverse-transcribed into complementary DNA using the Prime Script RT reagent kit (Takara, Shiga, Japan) according to the manufacturer's instructions. The expression of EBP50 was determined by real-time PCR using SYBR Premix Ex Taq (Takara, Shiga, Japan) and Step One Plus Real-Time PCR System (Applied Biosystems, CA). Primers used for PCR amplification were: GAPDH: 5-GCACCGTCAAGGCTGAGAAC-3' (forward), 5'-TGGTGAAGACGCCAGTGGA-3' (reverse); EBP50: 5'-AGGCCAGTTCATCCGAGCAG-3'(forward), 5'-CAGCAGCTTGGCCTCATCAC-3' (reverse). The mRNA levels of EBP50 relative to the control was calculated by $2^{-\mathrm{T}}$.

Western blotting. The cellular protein was collected and lysed using RIPA lysis buffer containing $1 \mathrm{mM}$ phen-ylmethylsulfonyl fluoride and $1 \%$ (vol/vol) protease inhibitor cocktail (Beyotime, Haimen, Jiangsu, China).Equal amounts of proteins were separated on sodium dodecyl sulfate polyacrylamide gel electrophoresis (SDS-PAGE) gels and transferred to polyvinylidene fluoride membranes at 4 for $1 \mathrm{~h}$. Membranes were blocked in 5\% BSA and incubated with primary antibodies against EBP50, LC3, Caspase-3, $\beta$-catenin, Ecadherin, Akt, MMP-2 and MMP-9 at 4 overnight. Then membranes were incubated at room temperature for $1 \mathrm{~h}$ with relevant secondary antibodies, and blots were visualized using enhanced chemiluminescence (ECL; Thermo Pierce, Rockford, Illinois, USA) according to the manufacturer's instructions and quantified by densitometry using Quantity One image software with $\beta$-actin used as an internal control.

MTT cytotoxicity assay. FLS were cultured in DMEM with $10 \%$ heat-inactivated FBS and antibiotics at $37^{\circ} \mathrm{C}$ in a humidifified atmosphere with $5 \% \mathrm{CO} 2.5 \times 10^{3}$ cells/well were plated in a 96-well plate. After 12 h, $20 \mu \mathrm{L}$ MTT solution $(0.5 \mathrm{mg} / \mathrm{mL})$ was added in FLS culture mediumand incubated for $4 \mathrm{~h}$. The culture mediumand in each well was discarded and added $150 \mu \mathrm{L}$ DMSO. The absorption of each well was measured at $490 \mathrm{~nm}$.

Phagocytosis assay by flow cytometry (FCM). FLS transduced with or without recombinant lentivirus for $48 \mathrm{~h}, 72 \mathrm{~h}, 96 \mathrm{~h}$. Then cells were washed thoroughly with D-Hanks buffer to remove extracellular recombinant lentivirus and depleted media, at the same time, fls were transfered seedind in 96 -well plates $5 \times 10^{3} /$ cells with annexin $\mathrm{V}$ binding buffer resuspended. Then were incubated at 2-8 for 15 min with $5 \mu$ l Annexin V-FITC, and were incubated at 2-8 for 5 min with $5 \mu \mathrm{l} \mathrm{PI}$, finally cells were collected and analyzed using Cytomics FC500 flow cytometer (Beckman Coulter Inc., Brea, CA, USA).

In Vitro Migration and Invasion Assay of FLS. In vitro cell migration assays were performed as described previously using Trans-well chambers $(8 \mu \mathrm{M}$ pore size,Corning, NY, USA). When cells reached subconfluency $(75 \%-80 \%)$, cells were serum-starved for $24 \mathrm{~h}$. After detachment with trypsin, cells were washed with PBS and resuspended in serum-free medium. Next, $100 \mu \mathrm{L}$ of cell suspension $\left(2 \times 10^{5}\right.$ cells $\left./ \mathrm{mL}\right)$ was added to the upper chamber while complete medium was added to the bottom wells. $24 \mathrm{~h}$ later, cells that had not migrated were removed from the upper surface of the filters using cotton swabs. Cells that had migrated were fixed with $5 \%$ glutaraldehyde solution to determine the number of migratory cells. The lower surface of the filters was stained with crystal violet. Images of three fields were captured from each membrane, and the number of migratory cells were counted. The mean of triplicate assays for each experimental condition was used.

Statistical analysis. All the presented data and results were confirmed in at least three independent 
experiments. Unpaired Student's t test or one-way analysis of variance was used to determine the significance of theresults. Data were considered statistically significant at $\mathrm{P}<0.05$.

\section{Results}

\section{Overexpression of EBP50 Suppresses the proliferation of FLS}

Inhibiting abnormal proliferation and activation of FLS may be a potentially therapeutic strategy to block or delay the progression of RA. To investigate the potential effects of EBP50 on the proliferation of FLS, fls were transfected with LV-EBP50 or LV-Plvx for $72 \mathrm{~h}$, then analyzed for the EBP50 expression and the proliferation of FLS. The results showed that LV-EBP50 transfection significantly increased the expression of EBP50 in both mRNA levels (Fig. 1A) and protein levels (Fig. 1B-1C), and MTT assay demonstrated that overexpression of EBP50 can suppresse the proliferation of FLS significantly, when compared with untransfected cells and LV-Plvx transfected cells (Fig. 1D). It has been reported that EBP50 can inhibit the proliferation of breast cancer cells by stimulating the autophagic lysosomal degradation, so we speculate whether EBP50 inhibits FLS cell proliferation by inhibiting autophagy. Then, the expression of LC3 was measured by western blotting in LV-EBP50 and LV-plvx transfected cells and untreated cells.As shown in (Fig. 1E-1F), the expressions of LC3 in FLS remain unchanged after transfected with LV-EBP50.Collectively, these results suggest that overexpression of EBP50 suppresses the proliferation of FLS and has no influence on autophagy.

\section{Overexpression of EBP50 promote apotosis of FLS By activating caspase-3}

FLS can exhibit tumor-like behavior, such as significant inhibition of apoptosis. As the major tumor suppressor genes, related literature reports have indicated that overexpression of EBP50 promoted significantly the tumor cells apotosis, and our previous results have showed that the overexpression of EBP50 upregulates the apoptosis ratio of macrophage by increasing the expression of Bax and caspase-3 in an NO-dependent mechanism. To investigated whether EBP50 can perform the same function in FLS, FLS were transfected with LV-EBP50 and LV-Plvx at the MOIs of 10 for $48 \mathrm{~h}, 72 \mathrm{~h}, 96 \mathrm{~h}$, and the cell apoptosis of FLS was analyzed by FCM. Results showed that EBP50 overexpression increased distinctly the apoptosis of FLS when compared with LV-Plvx transduced FLS $(\mathrm{P}<0.001)$ (Fig. 2A); The expression of apoptosis-related protein caspase-3 in FLS were measured by western blotting. As shown in (Fig. 2B), when FLS were transduced with LV-EBP50 and LV-Plvx for $96 \mathrm{~h}$, the protein expressions level of cleaved caspase-3 were significantly increased .

\section{overexpression of EBP50Suppresses the Migration and Invasion of FLS}

\section{By downregulating MMP-9}

Related researchs have showed that EBP50 could inhibit the migration and invasion of breast cancer cells by down-regulating MMPs and interacting with $\beta$-catenin(30,33). Since FLS migration and invasion are essential steps leading to joint destruction of RA, we evaluated the effect of EBP50 on these aggressive phenotypes of FLS in vitro. The primary FLS were left untreated (CON) or transfected with LV-EBP50 or LV-Plvx for $72 \mathrm{~h}$, then the migration and invasion of FLS were evaluated using the transwell assay. FLS overexpressing EBP50 displayed dramatic reduction in the number of migrating and invading cells across transwell assay (Fig. 3A-3B). As we known, the FLS could express MMPs and a variety of surface adhesion molecules to influence the migration and invasion. To study the potential mechanisms underlying the biological effect of EBP50 in FLS, the expression of $\beta$-catenin, E-cadherin, MMP2 and MMP9 were analyzed in LV-EBP50 and LV-Plvx transfected cells and untreated cells. The results have showed that protein levels of $\beta$-catenin were unchanged and E-cadherin were not expressed (Fig. 3C-3D), and the expression of MMP-9 in the EBP50-overexpressing cells were significantly decreased, whereas MMP-2 remained the same (Fig. 3E-3F$3 \mathrm{G}$ ). These results have indicated that overexpression of EBP50 impaired both the migratory and invasive ability of FLS by downregulating MMP-9.

\section{DISCUSSION}


So far, the pathogenesis of RA has not been completely clarified, therefore no therapeutic specific has been found clinically. The current treatment mainly focuses on drug therapy and surgical treatment. Current clinically used drugs include nonsteroidal anti-inflammatory drugs, glucocorticoid, disease modifying antirheumatic drugs, biologics, traditional Chinese medicine, etc. However, various therapies have their own limitations in the clinical treatment of RA. Recent studies have suggested genetic approach may offer new therapeutic methods, several genetics have been identified as genetic factors that contribute to RA, or have potential role in regulating the pathogenesis of RA, such as microRNAs ${ }^{[22,23]}$, $\operatorname{lncRNAs}{ }^{[24]}$, etc. There is growing evidence that FLS of active RA play a major role in the onset and progression of RA. In addition, FLS obtained from synovia of patients with RA exhibit tumour-like behaviours, including aggressive proliferation, increased migration, invasion, and reduced apoptosis. Emerging evidence indicate that inhibition of RA-FLS effector molecules might be beneficial for RA therapy. In this study, we expolred the role of overexpression of EBP50 in FLS proliferation, migration/invasion, and cell apoptosis.

There have many studies reported that EBP50 expression was down-regulated in breast cancer, gastric cancer and prostate cancer, and overexpression of EBP50 could effectively inhibit the proliferation of tumor cells and promote the apoptosis of tumor cells. Here in our study, to explore the potential effects of EBP50 on the proliferation of FLS, FLS were left untreated (CON) or transfected with control lentivirus (LVPlvx) or LV-EBP50 for $72 \mathrm{~h}$, the expression of EBP50 were detected by WB and qRT-PCR firstly. The results indicated that the expression of EBP50 was significantly up-regulated in LV-EBP50 transfected cells compared with untransfected cells and LV-Plvx transfected cells. Then, we found that EBP50 overexpression in FLS significantly inhibited cell proliferation by MTT assay, consistent with previous reports showing EBP50 as an inhibitor against cell proliferation in certain proliferative diseases such as cancer ${ }^{[25-27]}$. At the same time, EBP50 can stimulate autophagy and silencing EBP50 restrains it in cytoplasm ${ }^{[28]}$. EBP50, an activator of autophagy, causes lysosomal degradation of c-Myc to suppress the growth of MCF-7 breast cancer cells overexpressing ${ }^{[29]}$. Recent studies also have shown that in RA-FLS from RA patients or from animal models of RA, the autophagy pathway is persistently stimulated and apoptosis is downregulated, leading to synovial fibroblast proliferation and activation. So, we investigated the effect of EBP50 overexpression on autophagy in FLS. We observed that the autophagy-related proteins LC3 remain unchanged. Our results thus suggest that EBP50 overexpression had no effect on the autophagy of FLS, which might inhibit FLS proliferation through other pathways.

Apoptosis is another important target for anticancer therapy. Related literature reports that overexpression of EBP50 significantly promoted the tumor cells apotosis, and we found that overexpression of EBP50 upregulates the apoptosis ratio of macrophage by increasing the expression of Bax and caspase-3 in an NOdependent mechanism in a previous study ${ }^{[30]}$. Thus, we investigated whether EBP50 can perform the same function in FLS. It is well known that the Bcl-2 and caspase families play a vital role in cell survival/death. The Bcl-2 family can be functionally divided into antiapoptotic members (Bcl-2, Bcl-XL, Bcl-w, and CED 9), and proapoptotic members (Bax, Bad, and Bid) ${ }^{[31,32]}$. Among the members of the caspase family, caspases-3, -6 , and -7 are the effector caspases involved in the execution of cells in response to apoptotic stimuli, and caspase-3, the most important terminal executive molecule in the process of apoptosis, activation will lead to irreversible apoptosis ${ }^{[33]}$. In this study, after transduced with LV-EBP50, the apoptosis of FLS were increased when compared with LV-Plvx transduced FLS (Fig. 4B). Furthermore, we evaluated the expression of the apoptosis-related proteins Caspase3 (Fig. 4B). The results showed that cleaved caspase-3 were significantly increased in LV-EBP50-transduced FLS, which revealed that EBP50 overexpression promote apotosis of FLS through activating caspase-3. Our results demonstrated EBP50 transient overexpression significantly increased RA-FLS apoptosis, and therefore, may offer novel approaches against synovial hyperplasia.

FLS is the major cell population involved in the development of RA in synovial tissues. Previous studies have shown that FLS migrate and invade the cartilage and bone, contributing to pannus formation and tissue damage during RA progression. Regulation of FLS migration and invasion may be a new therapeutic strategy to prevent the destructive progress of RA. Prior studies indicated that the suppression of FLS migration and invasion might protect against joint destruction in RA. Therefore, we determined the effect of overexpression of EBP50 on the migration and invasion of FLS in this study. As a result, transfection of FLS 
with the LV-EBP50 over-expression vector had a profound inhibitory effect on their migration and invasion. It is well documented that membrane-associated proteins formed by the combination of E-cadherin and $\beta$ catenin participate in the regulation of cell adhesion, and cell movement, including metastasis and invasion, is also regulated by membrane-associated adhesion proteins. EBP50 stabilizes $\beta$-catenin on the cell surface by interacting with $\beta$-catenin and promotes the binding of $\beta$-catenin to E-cadherin complex, inhibiting cell migration and thus tumor cell metastasis. In addition, Matrix metalloproteinases (MMPs) are the main proteases responsible for the invasion and degradation of basement membranes and extracellular matrix. MMP-1, MMP-3, MMP-9, and MMP-13 are markedly elevated in synovial fluid from RA patients and play a significant role in collagen degradation, causing disease progression and bone erosion ${ }^{[34]}$. Among these proteins, endogenous MMP2 and MMP9 contribute to synovial fibroblast survival, proliferation, migration, and invasion in RA ${ }^{[35]}$. Studies have shown that EBP50 can down-regulate the expression of MMPs in breast cancer cells, especially MMP-2 and MMP-9, thus inhibiting the invasion ability of breast cancer cells, indicating that EBP50 can inhibit the invasion of tumor cells by inhibiting the expression of MMPs. To determine the effect of EBP50 on E-cadherin, $\beta$-catenin, MMP2 and MMP9 expression of FLS, we used western blot to measure the expression of E-cadherin, $\beta$-catenin, MMP2 and MMP9. In the present study, we observed that EBP50 overexpression markedly down-regulated the expression of MMP-9, but had no effect on MMP-2, E-cadherin and $\beta$-catenin, suggesting that one of the mechanisms for EBP50-regulated aggressive behavior of FLS is reducing MMP-9 production.

\section{Conclusions}

To summarize, EBP50 suppressed FLS proliferation, migration and invasion, and induced apoptosis. These findings have therapeutic significance and indicate the potential of EBP50 as a therapeutic target for RA, because the EBP50-mediated effects on FLS involve targeting synovial hyperplasia. Further research is needed to investigate the pathways involved in these protective effects and clarify if EBP50 could reduce proinflammatory cytokine secretion from RA-FLS.

\section{Acknowledgements}

This work was supported by the National Natural Science Foundation of China (81660277), Jiangxi Provincial Natural Science Foundation of China (20202BAB216001), the Science and Technology Project of Health and Family Planning Commission of Jiangxi Provinceof China (20203091).

\section{Disclosure}

The authors declare no conflicts of interest.

\section{References}

[1] Charlotte Hua, Claire I Daien, Bernard Combe, et al. Diagnosis, prognosis and classification of early arthritis: results of a systematic review informing the 2016 update of the EULAR recommendations for the management of early arthritis[J]. RMD Open. 2017;3(1):e000406.

[2] Josef S Smolen, Robert B M Landewé, Johannes W J Bijlsma, et al. EULAR recommendations for the management of rheumatoid arthritis with synthetic and biological disease-modifying antirheumatic drugs: 2019 update[J].Ann Rheum Dis. 2020;79(6):685-699.

[3] Buch MH, Emery P. New therapies in the management of rheumatoid arthritis[J]. Curr Opin Rheumatol.2011;23(3):245-251.

[4] Chan AC, Carter PJ. Therapeutic antibodies for autoimmunity and inflammation[J]. Nat Rev Immunol.2010;10(5):301-316.

[5] Gary Firestein, MD, Iain B McInnes FRCP PhD2. Immunopathogenesis of rheumatoid arthritis[J]. Immunity. 2017;46(2):183-196.

[6] Bartok B, Firestein GS. Fibroblast-like synoviocytes: key effector cells in rheumatoid arthritis[J].Immunol Rev.2010;233(1):233-255. 
[7] Iwanaga T, Shikichi M, Kitamura H, et al. Morphology and functional roles of synoviocytes in the joint[J]. Arch Histol Cytol,2000; 63(1):17-31.

[8] Chang SK, Gu Z,Brenner MB. Fibroblast-like synoviocytes in inflammatory arthritis pathology: the emerging role of cadherin-11[J]. Immunol Rev.2010;233(1):256-266.

[9] McInnes IB, Schett G. Cytokines in the pathogenesis of rheumatoid arthritis[J]. Nat Rev Immunol.2007;7(6):429-442.

[10] Hu F, Liu H, Xu L, et al. Hypoxia-inducible factor-1 $\alpha$ perpetuates synovial fibroblast interactions with $\mathrm{T}$ cells and B cells in rheumatoid arthritis[J]. Eur J Immunol.2016;46(3):742-751.

[11] García S, Liz M, Gómez-Reino JJ, et al. Akt activity protects rheumatoid synovial fibroblasts from Fas-induced apoptosis by inhibition of Bid cleavage[J]. Arthritis Res Ther.2010;12(1):R33.

[12] Kim SK, Park KY, Yoon WC, et al. Melittin enhances apoptosis through suppression of IL-6/sIL-6R complex-induced NF- $x$ B and STAT3 activation and Bcl-2 expression for human fibroblast-like synoviocytes in rheumatoid arthritis[J]. Joint Bone Spine.2011;78(5):471-477.

[13] Hsiao HB, Hsieh CC, Wu JB, et al. Kinsenoside inhibits the inflammatory mediator release in a type-II collagen induced arthritis mouse model by regulating the $\mathrm{T}$ cells responses[J]. BMC Complement Altern Med.2016;16(1):80.

[14] Mu N, Gu J, Huang T, et al. A novel NF- $\varkappa$ B/YY1/microRNA-10a regulatory circuit in fibroblast-like synoviocytes regulates inflammation in rheumatoid arthritis[J]. Sci Rep.2016;6:20059.

[15] Shang CH,Zhang QQ,Zhou JH.Oridonin Inhibits Cell Proliferation and Induces Apoptosis in Rheumatoid Arthritis Fibroblast-Like Synoviocytes[J]. Inflammation. 2016 ;39(2):873-80.

[16] Zou S, Wang C, Cui Z, et al. $\beta$-Elemene induces apoptosis of human rheumatoid arthritis fibroblast-like synoviocytes via reactive oxygen species-dependent activation of p38 mitogen-activated protein kinase[J]. Pharmacol Rep.2016;68(1):7-11.

[17] Yao W, Feng D, Bian W, et al. EBP50 inhibits EGF-induced breast cancer cell proliferation by blocking EGFR phosphorylation[J]. Amino Acids.2012;43(5):2027-2035.

[18] Lv XG, Ji MY, Dong WG, et al. EBP50 gene transfection promotes 5-fluorouracil-induced apoptosis in gastric cancer cells through Bax- and Bcl-2-triggered mitochondrial pathways $[\mathrm{J}]$. Mol Med Rep.2012;5(5):1220-1226.

[19] Bartholow TL, Becich MJ, Chandran UR, et al. Immunohistochemical analysis of ezrin-radixin-moesinbinding phosphoprotein 50 in prostatic adenocarcinoma[J]. BMC Urol.2011;11:12.

[20] Peng XL, Ji MY, Yang ZR, et al. Tumor suppressor function of ezrin-radixin-moesin-binding phosphoprotein-50 through $\beta$-catenin/E-cadherin pathway in human hepatocellular cancer[J]. World J Gastroenterol.2013;19(8):1306-1313.

[21] Li H, Zhang B, Liu Y, et al. EBP50 inhibits the migration and invasion of human breast cancer cells via LIMK/cofilin and the PI3K/Akt/mTOR/MMP signaling pathway[J]. Med Oncol.2014;31(9):162.

[22] Jie Wu, Wenqiang Fan, Ling Ma, et al. miR-708-5p promotes fibroblast-like synoviocytes' cell apoptosis and ameliorates rheumatoid arthritis by the inhibition of Wnt3a/ $\beta$-catenin pathway $[J]$. Drug Des Devel Ther. 2018;12:3439-3447.

[23] Jie Gao, Ruina Kong, Xiaoli Zhou, et al. MiRNA-126 expression inhibits IL-23R mediated TNF- $\alpha$ or IFN-rproduction in fibroblast-like synoviocytes in a mice model of collageninduced rheumatoid arthritis[J].Apoptosis. 2018;23(11-12):607-615. 
[24] Chun-Wang Zhang, Xia Wu, Dan Liu, et al. Long non-coding RNA PVT1 knockdown suppresses fibroblast-like synoviocyte inflammation and induces apoptosis in rheumatoid arthritis through demethylation of sirt6[J]. J Biol Eng. 2019;13:60.

[25] Yao W, Feng D, Bian W, et al. EBP50 inhibits EGF-induced breast cancer cell proliferation by blocking EGFR phosphorylation[J]. Amino Acids. 2012;43(5):2027-2035.

[26] Lv XG, Ji MY, Dong WG, et al. EBP50 gene transfection promotes 5-fluorouracil-induced apoptosis in gastric cancer cells through Bax- and Bcl-2-triggered mitochondrial pathways[J]. Mol Med Rep.2012;5(5):1220-1226.

[27] Bartholow TL, Becich MJ, Chandran UR, et al. Immunohistochemical analysis of ezrin-radixin-moesinbinding phosphoprotein 50 in prostatic adenocarcinoma[J]. BMC Urol.2011;11:12.

[28] Tang MK, Zhang JT. Caspase and its effects on apoptosis. Bull Med Res. 2000;

[29] Minxi Lao, Maohua Shi, Yaoyao Zou, et al. Protein inhibitor of activated STAT3 regulates migration, invasion, and activation of fibroblastlike synoviocytes in rheumatoid arthritis[J]. J Immunol. 2016;196(2):596606.

[30] Guo Y, Deng Y, Huang Z, et al. EBP50 induces apoptosis in macrophages by upregulating nitric oxide production to eliminate intracellular Mycobacterium tuberculosis[J]. Sci Rep.2016;6:18961.

[31] A. Pena-Blanco, A.J. Garcia-Saez. Bax, Bak and beyond - mitochondrial performance in apoptosis[J], FEBS J. 285 (3) (2018) 416-431.

[32] M.A. Elhinnawi, R.M. Mohareb, H.M. Rady, et al. Novel pregnenolone derivatives modulate apoptosis via Bcl-2 family genes in hepatocellular carcinoma in vitro[J]. Steroid Biochem. Mol. Biol. 183 (2018) $125-136$.

[33] Bao J, Wang S, Gunther LK, et al. The actin-bundling protein TRIOBP-4 and -5 promotes the motility of pancreatic cancer cells[J]. Cancer Lett. 2015;356(2 Pt B):367-73.

[34] Shan Zeng, Kefeng Wang, Mingcheng Huang, et al. Halofuginone inhibits TNF- $\alpha$-induced the migration and proliferation of fibroblast-like synoviocytes from rheumatoid arthritis patients[J]. Int Immunopharmacol. 2017;43:187-194.

[35] P Pratheeshkumar, Girija Kuttan. Andrographolide inhibits human umbilical vein endothelial cell invasion and migration by regulating MMP-2 and MMP-9 during angiogenesis[J]. J Environ Pathol Toxicol Oncol. 2011;30(1):33-41.

\section{Figure legends}

\section{Figure 1. Overexpression of EBP50 Suppresses the proliferation of FLS.}

FLS were left untreated (CON) or transfected with control lentivirus (LV-Plvx) or LV-EBP50 for 72 h.The mRNA levels of EBP50 were measured by real-time RT-PCR (A). The protein levels of EBP50 were detected by Western blotting (B).Band density of the specific protein was analyzed with Quantity One image software, and the results were expressed as average density to $\beta$-actin (C).FLS were left untreated (CON) or transfected with control lentivirus (LV-Plvx) or LV-EBP50 for $48 \mathrm{~h}$, Then were transfered seedind in 96-well plates $5 \times 10^{3} /$ cells, after night, $20 \mu \mathrm{L}$ MTT solution $(0.5 \mathrm{mg} / \mathrm{mL})$ was added in FLS culture mediumand incubated for $4 \mathrm{~h}$ in $5 \% \mathrm{CO} 2$ atmosphere at 37. The culture mediumand in each well was discarded and added 150 $\mu \mathrm{L}$ DMSO. The absorption of each well was measured at $490 \mathrm{~nm}$.(D). The protein levels of LC3 were detected by Western blotting (E).Band density of the specific protein was analyzed with Quantity One image software, and the results were expressed as average density to $\beta$-actin $(F)$.Data are represented as mean \pm SEM of at least three independent experiments. ${ }^{*} \mathrm{P}<0.05,{ }^{* *} \mathrm{P}<0.01,{ }^{* * *} \mathrm{P}<0.001$ compared with the uninfected group. 
Figure 2. Overexpression of EBP50 promote apotosis of FLS By activating caspase-3

FLS were left untreated (CON) or transfected with control lentivirus (LV-Plvx) or LV-EBP50 for 48 h,72 h,96 h.the apoptosis incidence was measured by $\mathrm{FCM}(\mathrm{A})$. The expression levels of caspase- 3 were determined by Western blotting(B). Data are represented as mean \pm SEM of at least three independent experiments. ${ }^{*} \mathrm{P}$ $<0.05,{ }^{* *} \mathrm{P}<0.01,{ }^{* * *} \mathrm{P}<0.001$ compared with the uninfected group.

Figure 3. overexpression of EBP50 Suppresses the Migration and Invasion of FLS

\section{By downregulating MMP-9}

FLS were left untreated (CON) or transfected with control lentivirus (LV-Plvx) or LV-EBP50 for $72 \mathrm{~h}$. Then the effect of EBP50 on migration was detected with transwell Boyden chamber, The images are representative of migration through the membrane after staining. Original magnifification $200 \times(A, C)$. The effect of EBP50 on migration was detected with transwell Boyden chamber, The images are representative of invasion through the membrane after staining. Original magnifification $200 \times(\mathrm{B}, \mathrm{D})$. The expression levels of $\beta$-catenin and Ecadherin were determined by Western blotting(E). Band density of the specific protein was analyzed with Quantity One image software (F). The expression levels of MMP-2 and MMP-9 were determined by Western blotting $(\mathrm{G})$. Band density of the specific protein was analyzed with Quantity One image software (H-I). Data are represented as mean $\pm \mathrm{SEM}$ of at least three independent experiments. ${ }^{*} \mathrm{P}<0.05,{ }^{* *} \mathrm{P}<0.01,{ }^{* * *} \mathrm{P}$ $<0.001$ compared with the uninfected group.

Figure 1. Overexpression of EBP50 Suppresses the proliferation of FLS.

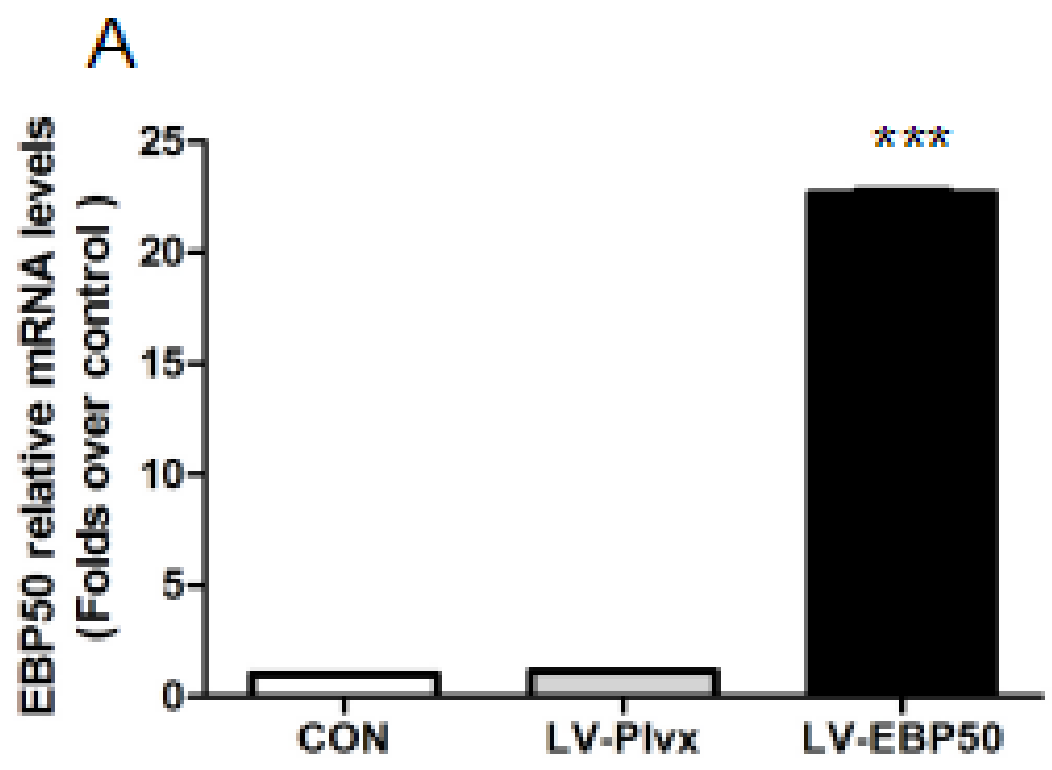


B

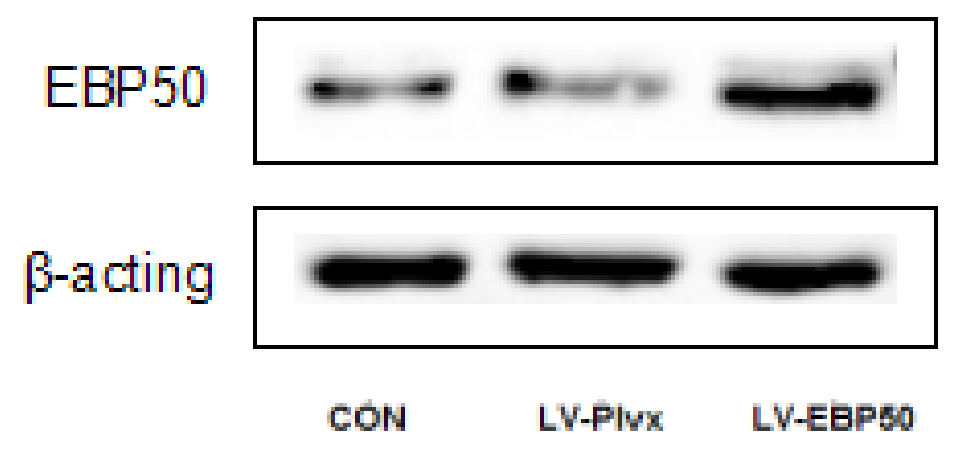

C

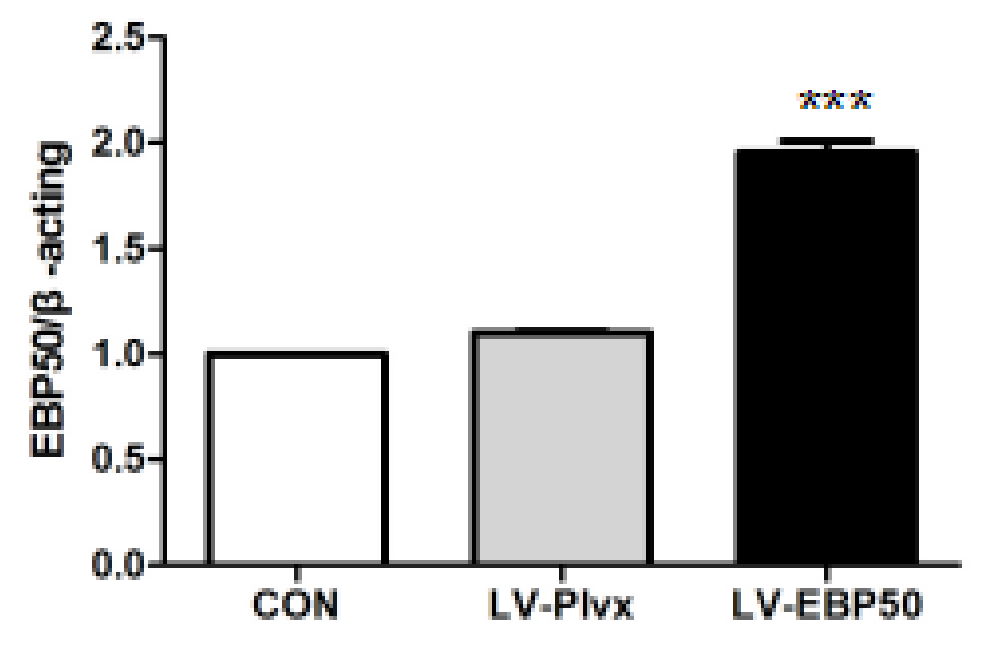


D

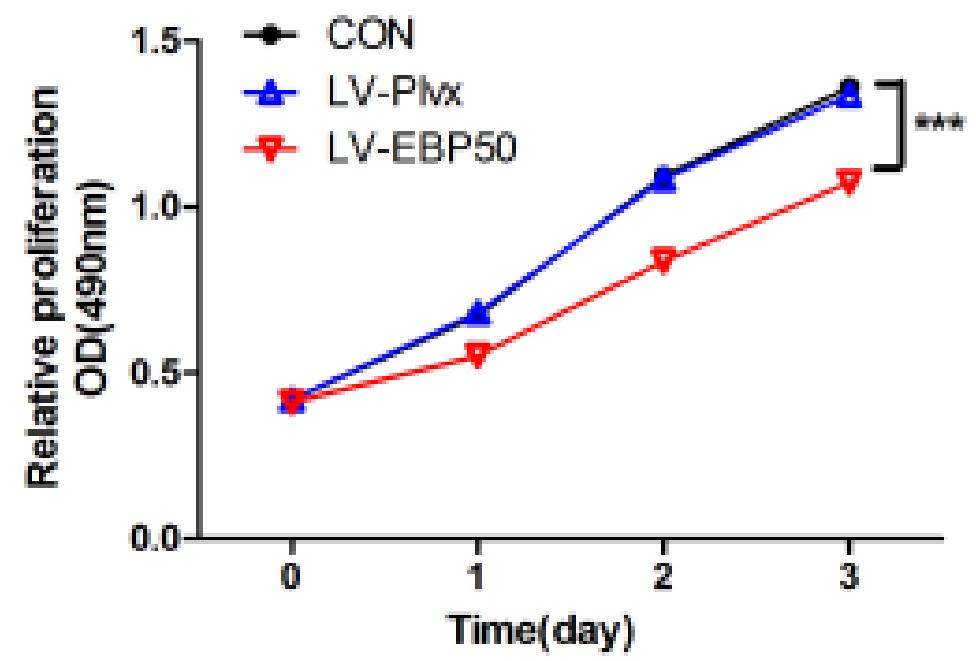

E
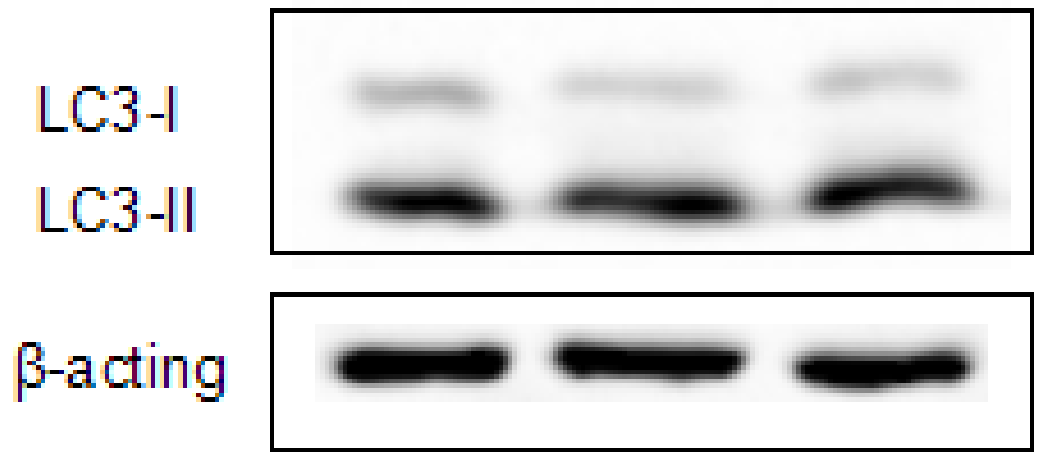

CON LY-PIVX LV-EBPSO 
F

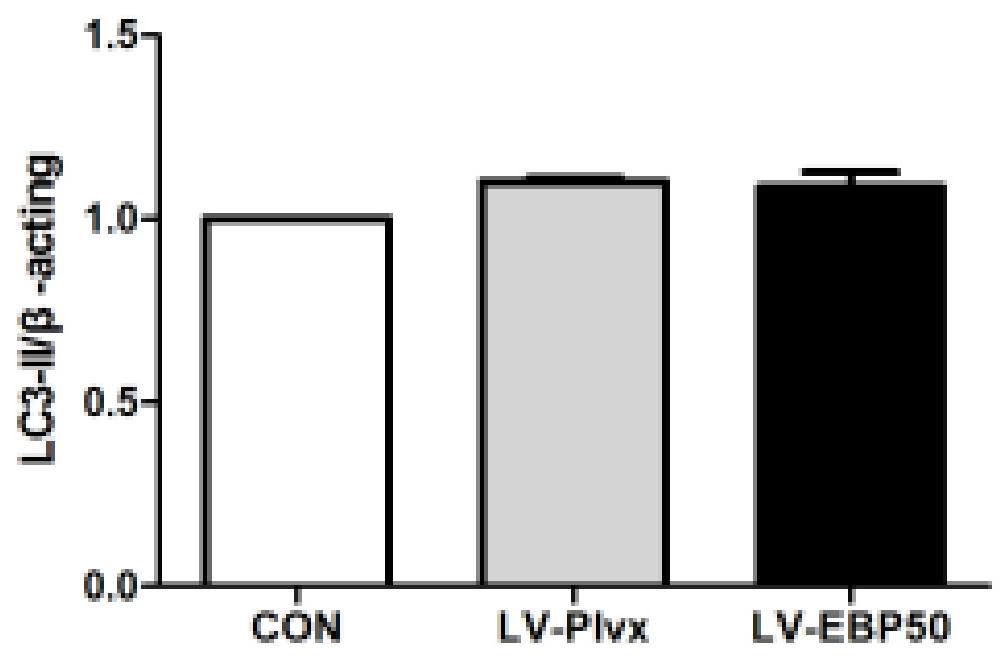

Figure 2. Overexpression of EBP50 promote apotosis of FLS By activating caspase-3

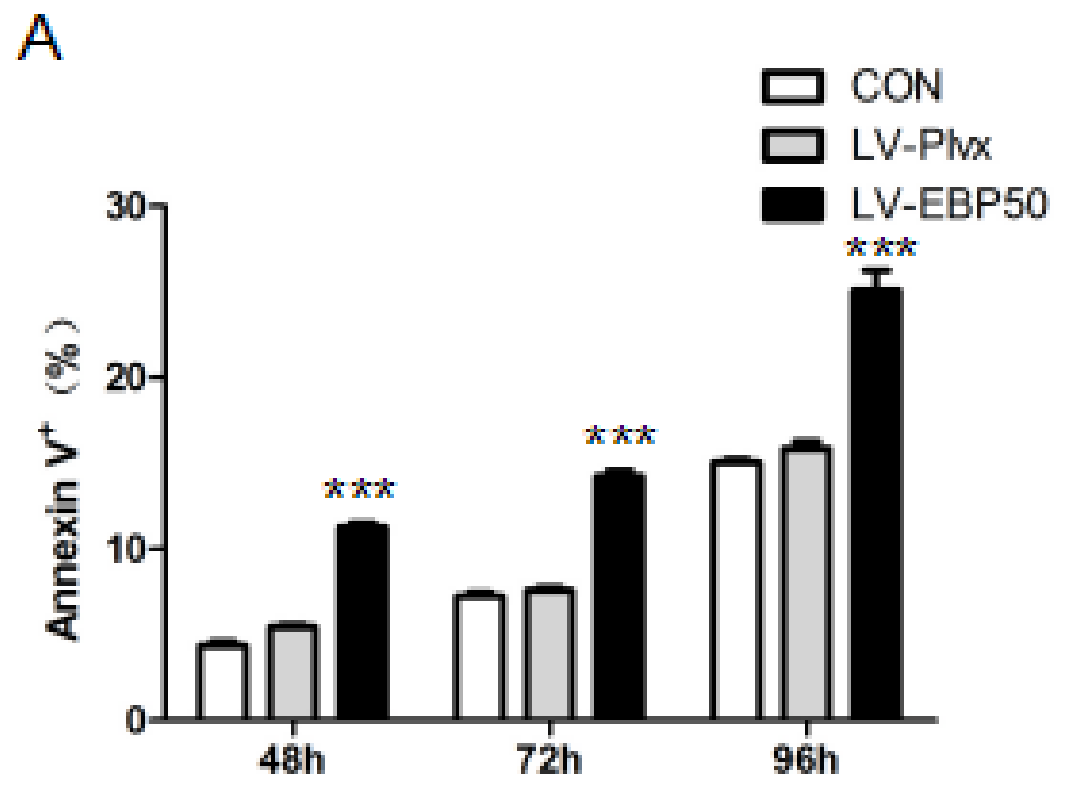


$\mathrm{B}$

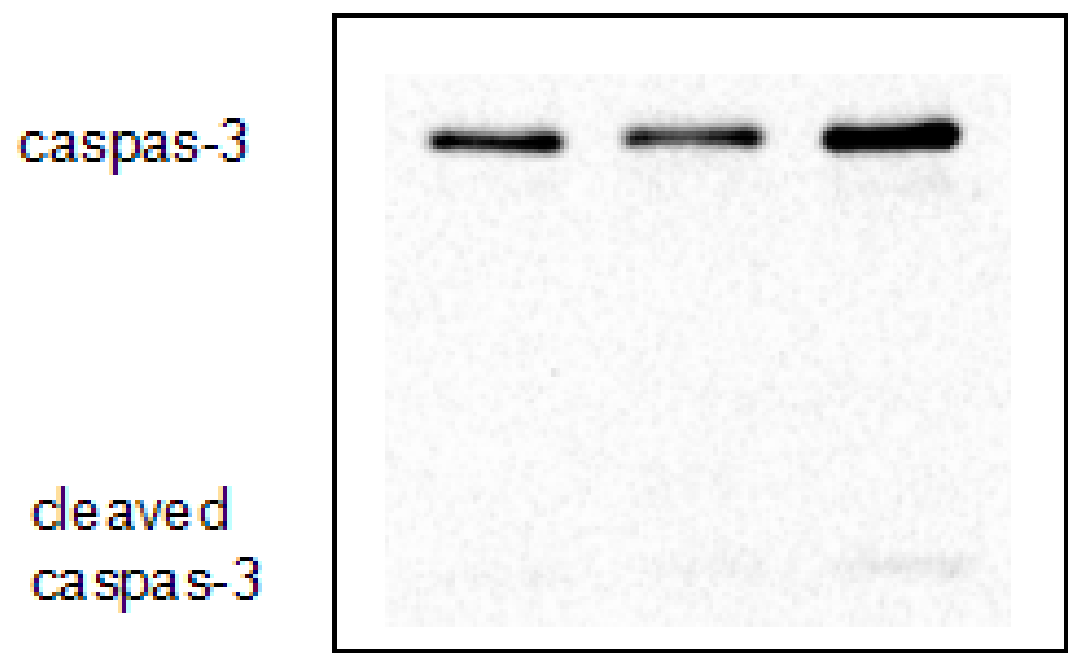

$\beta$-acting

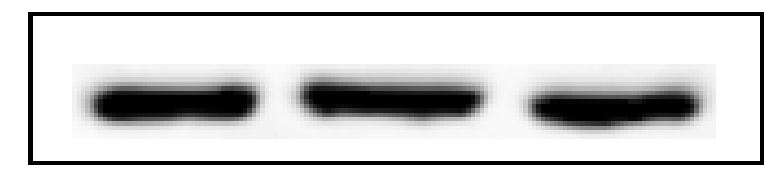

CON LV-PIV LV-EBPSO

Figure 3. overexpression of EBP50 Suppresses the Migration and Invasion of FLS By downregulating MMP-9

\begin{tabular}{llll}
\hline & CON & LV-Plvx & LV-EBP50 \\
$\mathrm{A}$ & & & \\
$\mathrm{B}$ & & & \\
\hline
\end{tabular}


C

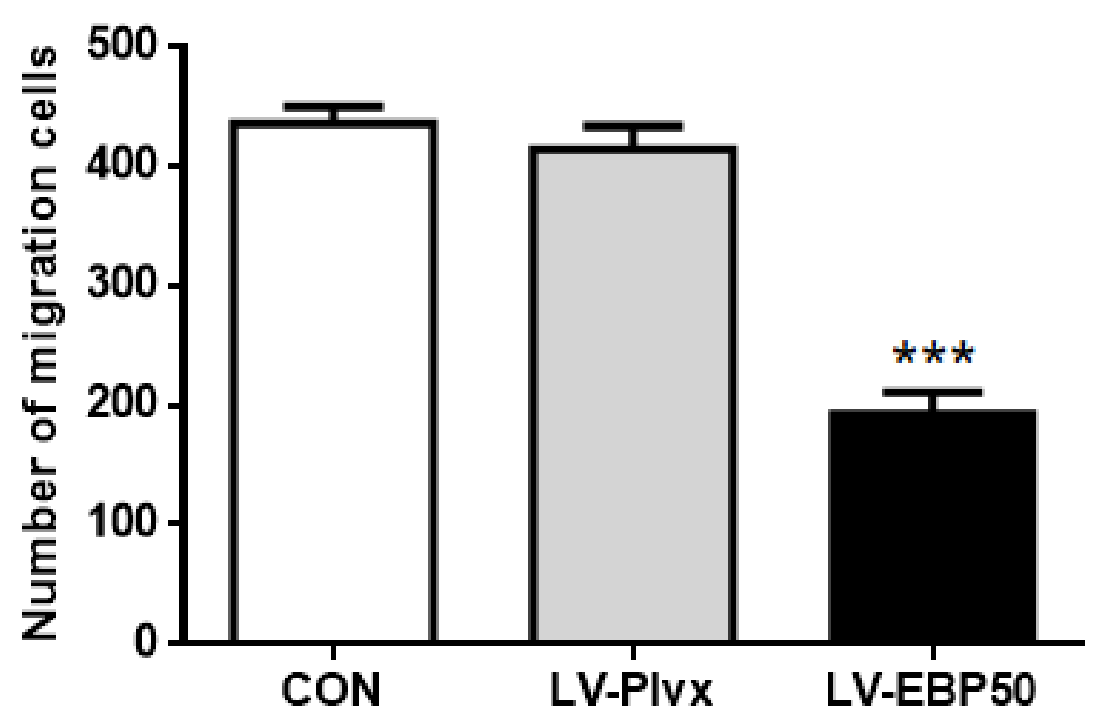

D

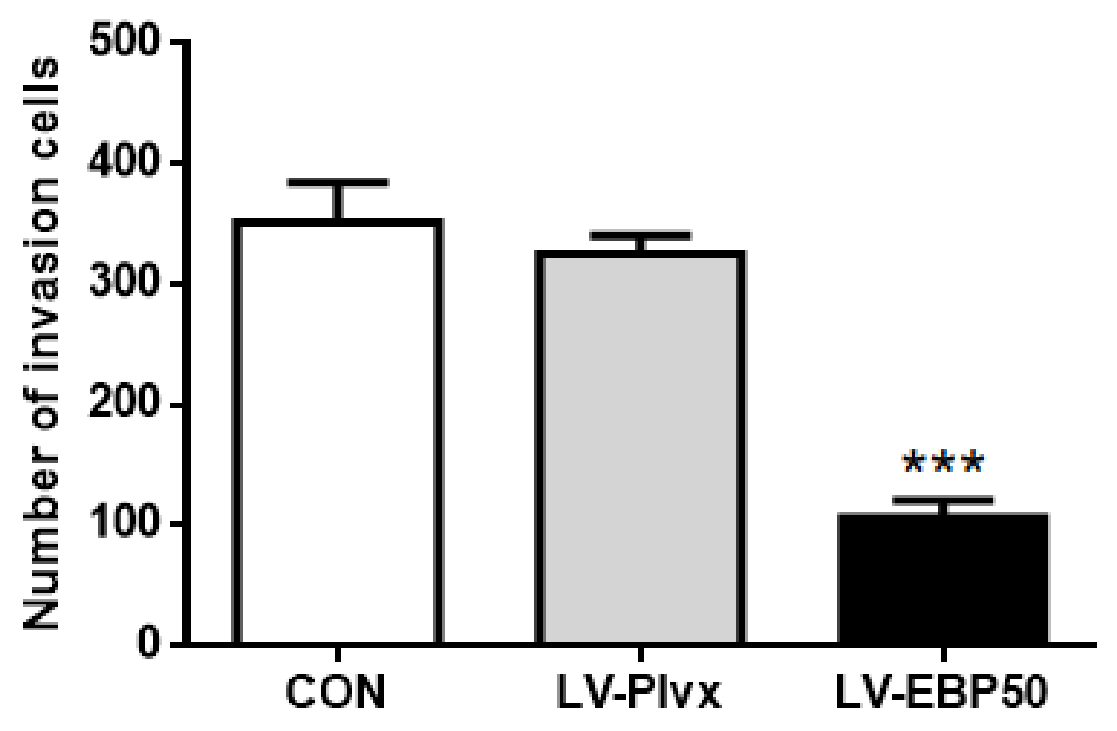




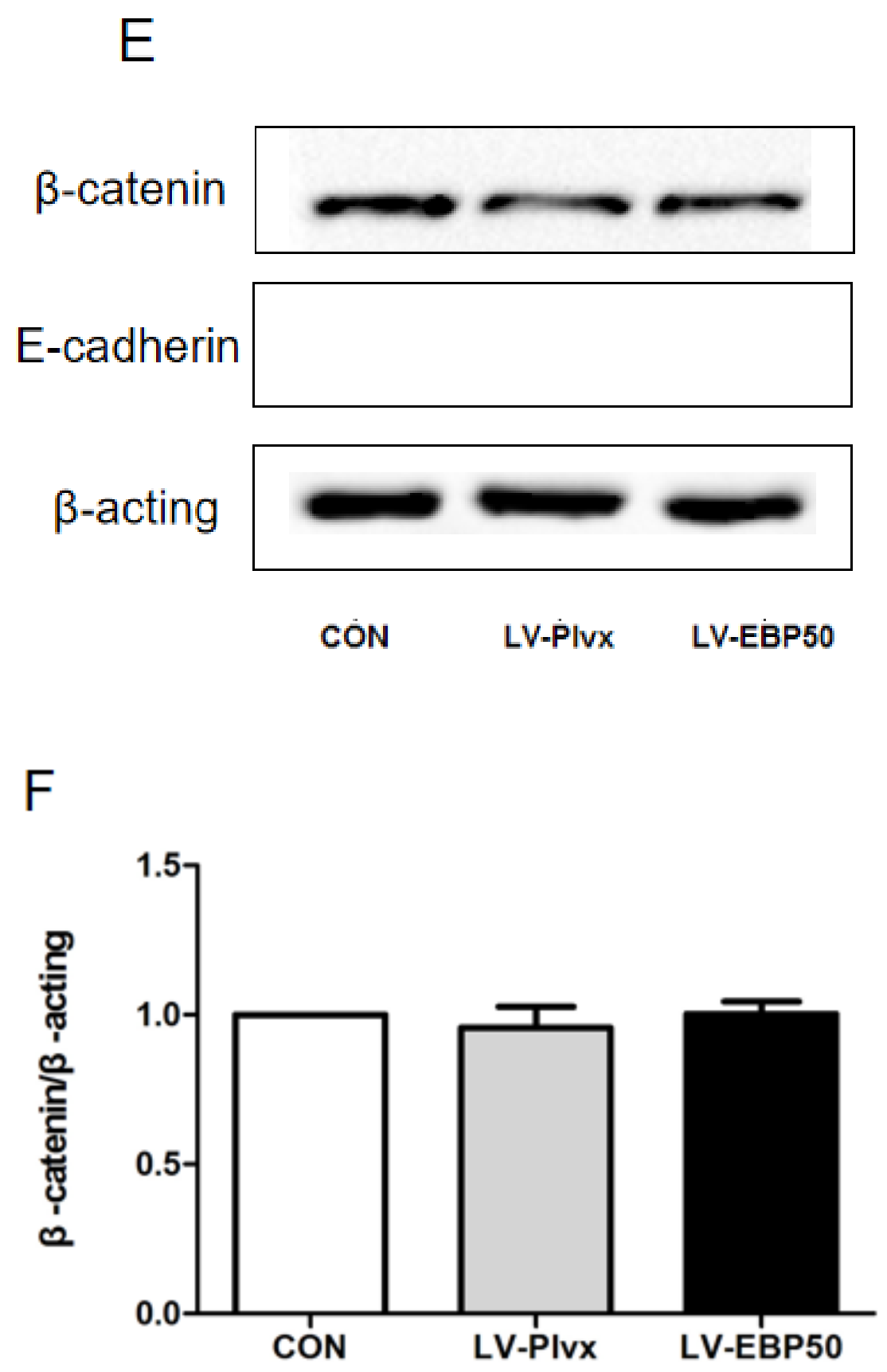


G

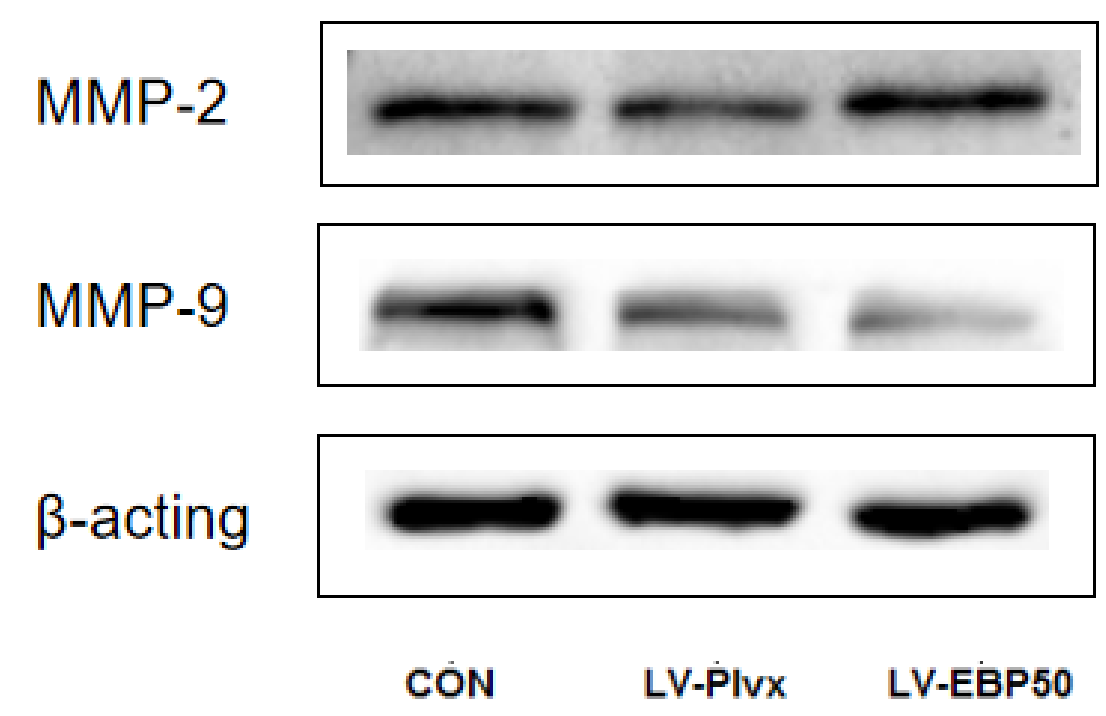

$\mathrm{H}$

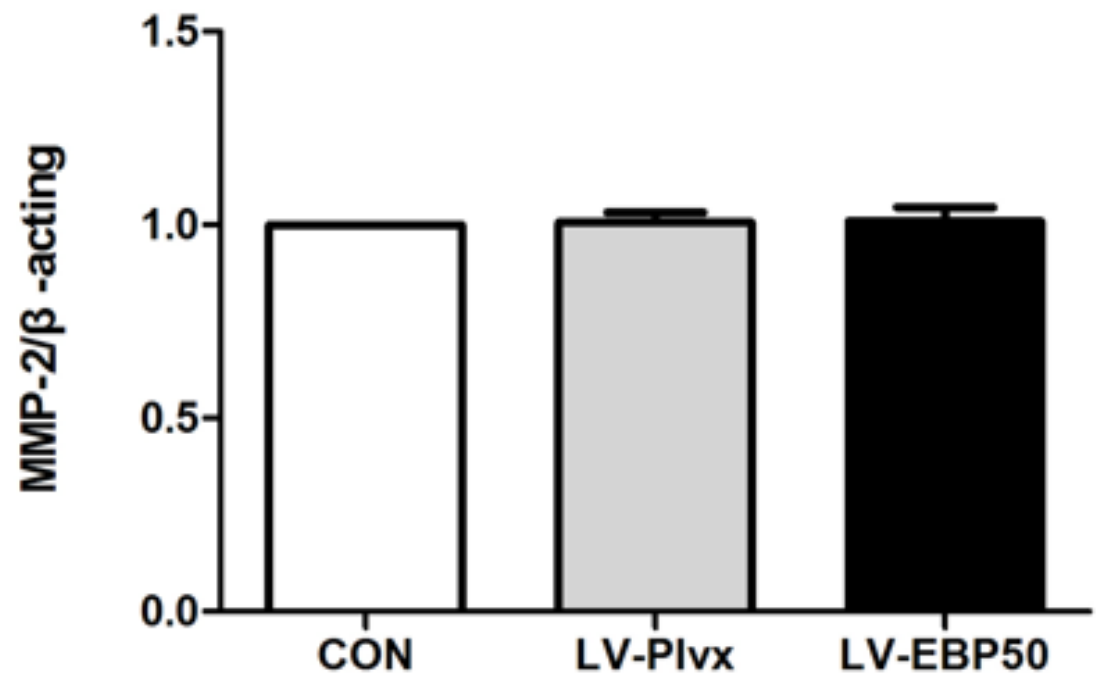




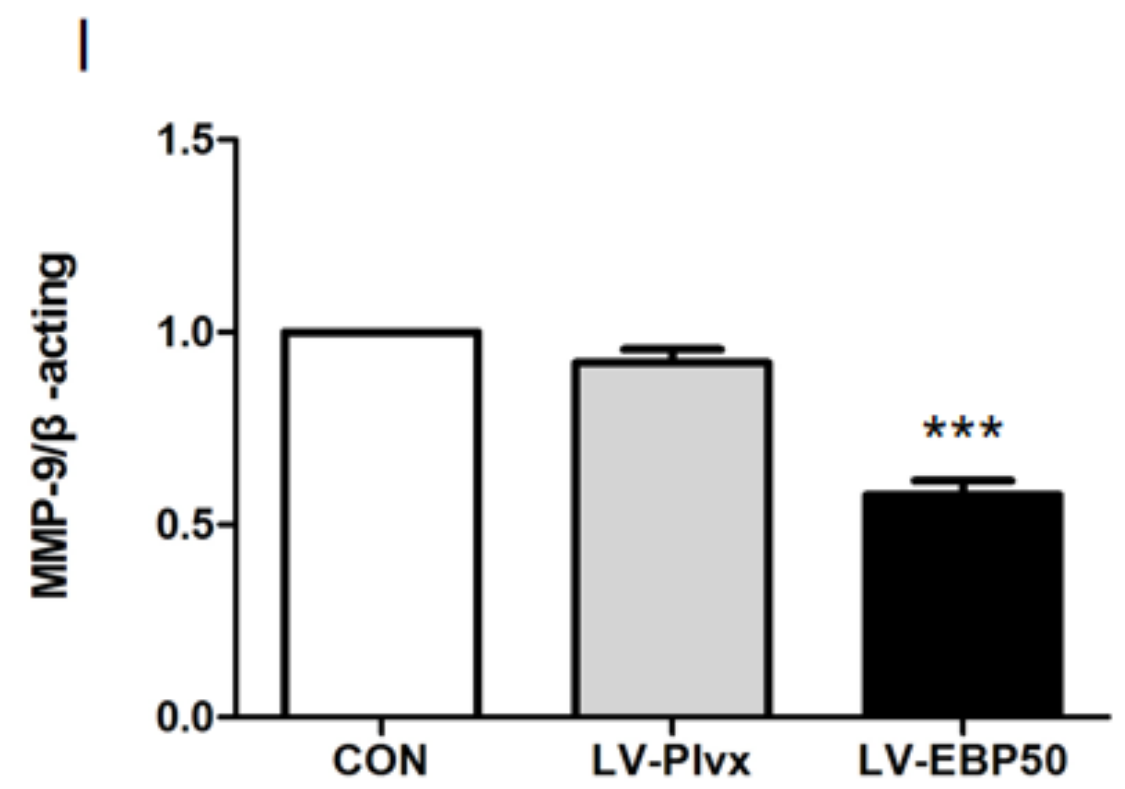

